\title{
Clinical Progress Note: High Flow Nasal Cannula Therapy for Bronchiolitis Outside the ICU in Infants
}

\author{
Laura Piper, MD ${ }^{1,2 *}$, Erika L Stalets, MD, MS ${ }^{1,3}$, Angela M Statile, MD, MEd'1,2,
}

'Department of Pediatrics, University of Cincinnati College of Medicine, Cincinnati, Ohio; ${ }^{2}$ Division of Hospital Medicine, Cincinnati Children's Hospital Medical Center, Cincinnati, Ohio; ${ }^{3}$ Division of Critical Care Medicine, Cincinnati Children's Hospital Medical Center, Cincinnati, Ohio; ${ }^{4}$ James M. Anderson Center for Health Systems Excellence, Cincinnati Children's Hospital Medical Center, Cincinnati, Ohio.

V iral bronchiolitis is the most common indication for infant hospitalization in the United States. ${ }^{1}$ The treatment mainstay remains supportive care, including supplemental oxygen when indicated. ${ }^{1}$ High flow nasal cannula (HFNC) therapy delivers humidified, heated air blended with oxygen, allowing much higher flow rates than standard nasal cannula therapy and is being used more frequently in inpatient settings.

\section{OVERVIEW AND CLINICAL QUESTION}

Infants and toddlers with bronchiolitis develop increased work of breathing to preserve oxygenation and ventilation in the setting of altered airway resistance and lung compliance. ${ }^{2,3}$ In addition to oxygen supplementation, HFNC is used to reduce work of breathing through several mechanisms:-26 (1) Nasopharyngeal dead space washout clears oxygen-depleted gas at the end of expiration, facilitating alveolar ventilation (ie, carbon dioxide retention improves); (2) High flow rates match increased inspiratory flow demands of acutely ill patients, reducing nasopharyngeal inspiratory resistance and optimizing dead space washout, thus decreasing work of breathing; (3) Adequate flow rates generate distending pressure, which prevents pharyngeal collapse, supports lung recruitment, and reduces respiratory effort (demonstrated in younger infants); and (4) HFNC systems heat and humidify the breathing gas, reducing the metabolic work required to condition cool, dry gas and improving conductance and pulmonary compliance. ${ }^{2-5}$

HFNC therapy is used more commonly in acute care units despite limited literature on its effectiveness outside the intensive care unit (ICU). ${ }^{7.8}$ We asked the question, "Does use of HFNC therapy for infants with bronchiolitis hospitalized in acute care units result in improved outcomes when compared with standard nasal cannula oxygen therapy, including length of stay (LOS), oxygen therapy duration, and preventing escalations of care such as ICU transfer, positive pressure ventilation, and intubation?" Also, do published studies provide guidance for the initiation and management of HFNC? We focused our search on studies published in the last five years that included patients

*Corresponding Author: Laura Piper, MD; E-mail: Laura.Piper@cchmc.org; Telephone: 513-803-5530

Published online first November 20, 2019

Received: May 10, 2019; Revised: September 6, 2019;

Accepted: September 11, 2019

๑ 2020 Society of Hospital Medicine DOI 10.12788/jhm.3328 with bronchiolitis treated with HFNC outside the ICU; here, we review those studies most relevant to pediatric hospitalists.

\section{RECENT LITERATURE REVIEW}

No guideline exists for initiating flow or fraction of inspired oxygen $\left(\mathrm{FiO}_{2}\right)$. $\mathrm{HFNC}$ may be initiated for hypoxia, increased work of breathing, or both in patients with bronchiolitis. To achieve optimal dead space washout, inspiratory flow, and distending pressure, initial flow rates should be 1.5 to $2 \mathrm{~L} / \mathrm{kg} / \mathrm{min}$, particularly for infants and young children. ${ }^{2.5}$ Weiler et al. ${ }^{3}$ evaluated the breathing effort of ICU patients at 0.5, 1, 1.5, and $2 \mathrm{~L} / \mathrm{kg} /$ min and found optimal flow rates for improved work of breathing were $1.5-2 \mathrm{~L} / \mathrm{kg} / \mathrm{min}$. The smallest patients, $\leq 8 \mathrm{~kg}$, saw the greatest benefit, a finding likely explained by larger anatomic dead space in infants/small children compared with older children. ${ }^{3}$ For older/larger children $(>20 \mathrm{~kg})$, an initial flow closer to $1 \mathrm{~L} / \mathrm{kg} / \mathrm{min}$ is often appropriate. ${ }^{5}$ When used for hypoxia, initiating flow without supplemental $\mathrm{FiO}_{2}$ may improve oxygenation by flushing nasopharyngeal dead space. $\mathrm{FiO}_{2}$ should be titrated to achieve the goal set by the treatment team, often $\geq 90 \%$. Improvement in heart rate and peripheral oxygen saturation $\left(\mathrm{SpO}_{2}\right)$ can be observed within 60 minutes of initiating HFNC in patients responsive to therapy. ${ }^{6}$

HFNC therapy is safe when used correctly. $6,9,10$ Potential adverse effects include pneumothorax, pressure injury, mucosal injury/bleeding, and delayed escalation to invasive ventilation. While difficult to quantify, recent studies report low rates or no serious HFNC complications. For example, only 2 of 1,127 patients supported with HFNC developed a pneumothorax and neither required evacuation. $2,9-12$

Inclusion criteria and HFNC protocols vary among published studies. Most HFNC protocols reviewed may not have optimally supported all of the patients in their HFNC groups, often by limiting flow to $<2 \mathrm{~L} / \mathrm{kg} / \mathrm{min}{ }^{6-9,11,12}$ These variables may explain the disparate results, with some studies demonstrating apparent benefits and others no difference. $.7,10,12$

Two studies of infants with bronchiolitis showed HFNC therapy may prevent ICU transfer, but this benefit may be limited to rescue when standard oxygen therapy fails, rather than as a superior initial support modality. ${ }^{7.9}$ Kepreotes et al. ${ }^{9}$ reported a single-center, randomized controlled trial comparing HFNC with standard oxygen therapy with 101 patients in each treatment arm. The primary outcome, median time to wean off oxygen, was not significantly different between the two groups: 24 hours (95\% Cl: 18-28) in the HFNC group 
TABLE. Comparison of Studies

\begin{tabular}{l} 
Study [Brief Details] \\
\hline Franklin et al. (2018)10 \\
Multicenter, randomized, controlled trial of early \\
HFNC treatment in infants with bronchiolitis \\
and hypoxemia in the ED and pediatric wards \\
compared to ST (low flow) \\
HFNC rate: 2 L/kg/min; Max Flow: None \\
$\mathrm{N}=1,472$ \\
733 in ST group \\
739 in HFNC group
\end{tabular}

Inclusion/Exclusion Criteria

Inclusion: $<12$ months with bronchiolitis

requiring supplemental $\mathrm{O}_{2}$ to keep $\mathrm{SpO}_{2} 92 \%$ 98\% (11 hospitals used $94 \%-98 \%$ )

Exclusion: critically ill needing respiratory support and ICU admission; cyanotic heart disease, basal skull fracture, upper airway obstruction, craniofacial malformation, home $\mathrm{O}_{2}$

\section{Outcome Measures}

$1^{\circ}$ : Escalation of care due to treatment failure, defined by meeting 3 of 4 clinical criteria: unchanged/increased $\mathrm{HR}$; unchanged/increased $\mathrm{RR} ; \mathrm{FiO}_{2}>0.4$ in HFNC group or $>2 \mathrm{LPM}$ $\mathrm{O}_{2}$ in ST group to maintain minimum $\mathrm{SpO}_{2}$; hospital early warning tool triggers review and escalation of care and clinicians determine

escalation required

$2^{\circ}$ : transfer to ICU, hospital LOS, ICU LOS, duration of $\mathrm{O}_{2}$ therapy, intubation rates, and adverse events

\section{$1^{\circ}$ : Time to wean off $\mathrm{O}_{2}$}

Inclusion: $<24$ months with moderate severity

\section{Kepreotes et al. (2017) ${ }^{9}$}

Single-center, randomized, controlled trial of HFNC compared to ST to examine whether HFNC provided enhanced respiratory support

HFNC rate: $1 \mathrm{~L} / \mathrm{kg} / \mathrm{min}$; Max Flow: $20 \mathrm{~L} / \mathrm{kg}$ $\mathrm{N}=202$

101 in ST group

101 in HFNC group bronchiolitis requiring supplemental $\mathrm{O}_{2}$

Exclusion: severe or life-threatening bronchiolitis (witnessed apnea, RR $>70$, moderate-severe grunting, $\mathrm{HR}>180$ or $<100, \mathrm{SpO}_{2}<90 \%$ on room air or $<92 \%$ on 2 LPM NC, post-ICU admission, transferred from other facility on $\mathrm{O}_{2}$ known diagnosis of asthma, pneumothorax, nasal trauma) adverse events, transfer to ICU, LOS, baseline- $2^{\circ}$ : Time to treatment failure, the proportion of treatment failure, the proportion of serious adjusted HR and RR at 4 and 24 hours

Parent-Reported (via follow-up phone call): delayed serious adverse events, subsequent medical care, concern with $\mathrm{O}_{2}$ therapy, and rating of the child's comfort, ability to feed, and sleep quality while on treatment

\section{RR and respiratory effort}

Ability to feed

Duration of $\mathrm{O}_{2}$ supplementation Hospital LOS
Results

Treatment failure with escalation of care: 87 of $739(12 \%)$ in HFNC group transferred to the ICU; 8 were intubated

167 of $733(23 \%)$ in ST group crossed over to HFNC; 102 rescued on HFNC, 65 failed rescue and transferred to the ICU; 4 were intubated

No difference in the hospital or ICU LOS, duration of $\mathrm{O}_{2}$ therapy; 1 pneumothorax/group

No life-threatening adverse events

Time to wean off $\mathrm{O}_{2}$ did not differ significantly between HFNC and ST arms

Treatment failure with escalation of care:

14 of $101(14 \%)$ in HFNC arm transferred to ICU 33 of $101(33 \%)$ in ST arm; 20 rescued on HFNC; 12 failed rescue and transferred to ICU

$90 \%$ of HFNC arm remained treatment failure-free at 24 hours vs $60 \%$ of ST arm

No serious adverse events, eg, pneumothorax

\section{Milani et al. (2016) ${ }^{12}$}

single-center observational study to compare vs ST for mod-severe bronchiolitis in infants

HFNC rate: $\mathrm{L} / \mathrm{min}=8 \mathrm{~mL} / \mathrm{kg}^{\text {* }}$ respiratory rate* 0.3

Max Flow: Not described

$\mathrm{N}=36$

18 in ST group

18 in HFNC group

\section{Riese et al. (2017)}

Single-center, retrospective, pre-post

intervention study assessing the association

of implementing a HFNC guideline on the

ward with clinical outcomes of infants with bronchiolitis

\section{HFNC rate: \\ $<6$ mos: $8 \mathrm{~L} / \mathrm{min}$ \\ 6-18 mos: $12 \mathrm{~L} / \mathrm{min}$ \\ 18-24 mos: $15 \mathrm{~L} / \mathrm{min}$ \\ Max Flow: Age dependent, $15 \mathrm{~L} / \mathrm{min}$ \\ $\mathrm{N}=1,937$}

936 before implementation; 1,001 after
Inclusion: $<12$ months with moderate or severe bronchiolitis requiring supplemental $\mathrm{O}_{2}\left(\mathrm{SpO}_{2}<\right.$

Exclusion: gestation age $<34$ weeks, admission to NICU at birth, history of bronchiolitis or wheezing episode, chronic respiratory disease, congenital airway anomalies, craniofacial malformations, hemodynamically significant heart disease, neurological disease, admission to the PICU

\section{Inclusion: charts with ICD-9 codes for} bronchiolitis, apnea, acute upper respiratory infection, or unspecified viral illness, and age $<24$ months

Exclusion: LOS $>21$ days, gestational age $<37$ weeks, chronic lung disease, asthma chromosomal abnormalities, heart disease, or neurological disease

Abbreviations: $\mathrm{ED}$, emergency department; $\mathrm{FiO}_{2}$, fraction of inspired oxygen; $\mathrm{HR}$, heart rate; HFNC, high flow nasal cannula; $\mathrm{HR}$, heart rate; ICD9, International Classification of Diseases, 9th edition; ICU, intensive care unit; LOS, length of stay; LPM, liters per minute; NC, nasal cannula; NICU, neonatal intensive care unit; PICU, pediatric intensive care unit; RR, respiratory rate; $\mathrm{SpO}_{2}$, peripheral capillary oxygen saturation; ST; standard therapy.

versus 20 hours in the standard therapy group $(95 \% \mathrm{Cl}: 17-$ 34). The HFNC group had fewer treatment failures (abnormal heart rate, respiratory rate, $\mathrm{SpO}_{2}<90 \%$, or severe respiratory distress score while on maximum therapy) than the standard therapy group, and 20 (63\%) of the 33 patients who failed standard therapy were rescued with HFNC, avoiding transfer to the ICU. Fourteen patients from the HFNC group and 12 from the standard oxygen group required transfer to the ICU for support escalation. Although this study did not show a significant difference in oxygen weaning time between groups, it appears to support HFNC use as a rescue modality to reduce or prevent ICU transfer. ${ }^{9}$ Franklin et al. ${ }^{10}$ conducted a multicenter, randomized, controlled trial to compare stan- dard nasal cannula oxygen therapy with HFNC (2 L/ $\mathrm{kg} / \mathrm{min}$ ) in 1,472 patients. Patients receiving HFNC had lower care escalation rates due to treatment failure, defined as the presence of at least three of four clinical criteria and the clinician determining escalation was indicated. Oxygen therapy duration, ICU admission rates, and LOS were not significantly different between groups. Similar to the previous study, a large portion of the standard therapy patients who failed treatment (102 of 167) crossed over to the HFNC arm in an attempt to avoid ICU transfer. Twelve patients required intubation: 8 (1\%) receiving HFNC and 4 (0.5\%) receiving the standard therapy. ${ }^{10}$

Two additional studies, both with study design limitations, did not demonstrate differences in ICU transfer rates and had 
variable differences in outcomes. Riese et al. ${ }^{7}$ retrospectively assessed HFNC use outside the ICU at one institution and included 936 patients admitted before and 1,001 patients admitted after HFNC guideline implementation on the wards. Flow rates were based on age and not weight. They found no difference in LOS, ICU transfer rate, ICU LOS, intubation rates, or 30-day readmission rates, though HFNC use increased over time. The HFNC guideline is a potentially significant limitation as it may not have provided optimal flow rates to all subjects given it was based on age rather than weight. Milani et al. ${ }^{12}$ performed a single-center observational study of 36 infants aged $<12$ months, treated for bronchiolitis on the ward, who were informally assigned to HFNC or standard therapy based upon HFNC device availability. HFNC flow rate was determined by the equation: $\mathrm{L} / \mathrm{min}=8 \mathrm{~mL} / \mathrm{kg} \times$ respiratory rate $\times$ 0.3. Using mean weight and respiratory rate for patients in this group, it appears patients in the HFNC group were treated with flow rates less than the $1.5-2 \mathrm{~L} / \mathrm{kg} / \mathrm{min}$ recommended to be effective. 2,3,12 Despite this, clinical improvement was faster in the HFNC group, including respiratory rate and effort, ability to feed, days on oxygen supplementation, and hospital LOS. ICU admission was not different between the two groups. ${ }^{12}$ The Table compares the four studies discussed above.

Given increasing use of HFNC outside the ICU, institutions risk overuse and increased healthcare costs. ${ }^{13}$ Limited data on HFNC overuse exist, but several studies report increased use after implementation on the wards without robust evidence indicating it improves outcomes. ${ }^{7,14}$ Overuse of HFNC is a concern that should be considered as institutions develop HFNC protocols. Another important consideration is safe feeding. One study examined 132 children ages one month to two years with bronchiolitis who were receiving HFNC and enteral nutrition. ${ }^{15}$ Only one patient had aspiration respiratory failure, and 12 had nutrition interruptions, demonstrating oral nutrition is generally well tolerated ${ }^{15}$ and should be considered in patients with stable respiratory status on HFNC.

\section{CONCLUSIONS}

Many children's hospitals have extended the use of HFNC outside the ICU for children with bronchiolitis despite the paucity of evidence demonstrating its benefit over standard flow oxygen. Given variation in protocols, study designs, outcomes, and number of patients studied, it is difficult to assess its efficacy outside the ICU. However, based on the studies reviewed herein, HFNC therapy does not appear to decrease LOS, time on oxygen, or escalations of care, such as ICU transfers, positive pressure ventilation, or intubation, when used as a primary therapy. ${ }^{7,911,12}$ Future research will ideally use optimal flow rates to determine the effectiveness of HFNC on acute care units. Although not addressed in the above studies, additional benefits to be considered in future studies include: (1) increased critical care capacity by allowing patients to be supported on the floor and (2) the ability for patients to remain closer to home when HFNC is used in the community hospital setting.

In each of the large, randomized studies reviewed, most (66\%-75\%) patients treated with standard low flow oxygen were supported successfully and did not require escalation to HFNC. 9,10 Hospitalists should continue to use standard low flow oxygen as first-line respiratory support for patients with bronchiolitis. ${ }^{1}$ No evidence supports the use of HFNC therapy early in a child's inpatient course; rather, it should be used when standard oxygen therapy fails. Future research should focus on better elucidating which patients will benefit most from HFNC to prevent overuse.

Disclosures: The authors have no financial relationships relevant to this article to disclose.

\section{References}

1. Ralston SL, Lieberthal AS, Meissner HC, et al. Clinical practice guideline: the diagnosis, management, and prevention of bronchiolitis. Pediatrics. 2014;134(5):e1474-1502. https://doi.org/10.1542/peds.2014-2742.

2. Milesi $\mathrm{C}$, Baleine J, Matecki S, et al. Is treatment with a high flow nasal cannula effective in acute viral bronchiolitis? A physiologic study. Intensive Care Med. 2013;39(6):1088-1094. https://doi.org/10.1007/s00134-013-2879-y.

3. Weiler T, Kamerkar A, Hotz J, Ross PA, Newth CJL, Khemani RG. The relationship between high flow nasal cannula flow rate and effort of breathing in children. J Pediatr. 2017;189:66-71. https://doi.org/10.1016/j.jpeds.2017.06.006.

4. Dysart K, Miller TL, Wolfson MR, Shaffer TH. Research in high flow therapy: mechanisms of action. Respir Med. 2009;103(10):1400-1405. https://doi. org/10.1016/j.rmed.2009.04.007

5. Milesi C, Boubal M, Jacquot A, et al. High-flow nasal cannula: recommendations for daily practice in pediatrics. Ann Intensive Care. 2014;4(1):29. https:// doi.org/10.1186/s13613-014-0029-5

6. Heikkila P, Sokuri P, Mecklin M, et al. Using high-flow nasal cannulas for infants with bronchiolitis admitted to paediatric wards is safe and feasible. Acta Paediatr. 2018;107(11):1971-1976. https://doi.org/10.1111/apa.14421.

7. Riese J, Porter T, Fierce J, Riese A, Richardson T, Alverson BK. Clinical outcomes of bronchiolitis after implementation of a general ward high flow nasal cannula guideline. Hosp Pediatr. 2017;7(4):197-203. https://doi.org/10.1542/ hpeds.2016-0195.

8. Betters KA, Gillespie SE, Miller J, Kotzbauer D, Hebbar KB. High flow nasal cannula use outside of the ICU; factors associated with failure. Pediatr Pulmonol. 2017;52(6):806-812. https://doi.org/10.1002/ppul.23626.

9. Kepreotes E, Whitehead B, Attia J, et al. High-flow warm humidified oxygen versus standard low-flow nasal cannula oxygen for moderate bronchiolitis (HFWHO RCT): an open, phase 4, randomised controlled trial. Lancet. 2017;389(10072):930-939. https://doi.org/10.1016/S0140-6736(17)30061-2.

10. Franklin D, Babl FE, Schibler A. High-flow oxygen therapy in infants with bronchiolitis. N Engl J Med. 2018;378(25):2446-2447. https://doi.org/10.1056/ NEJMc1805312

11. Mayfield S, Bogossian F, O'Malley L, Schibler A. High-flow nasal cannula oxygen therapy for infants with bronchiolitis: pilot study. J Paediatr Child Health. 2014;50(5):373-378. https://doi.org/10.1111/jpc.12509.

12. Milani GP, Plebani AM, Arturi $E$, et al. Using a high-flow nasal cannula provided superior results to low-flow oxygen delivery in moderate to severe bronchiolitis. Acta Paediatr. 2016;105(8):e368-e372. https://doi.org/10.1111/ apa.13444.

13. Modesto i Alapont V, Garcia Cusco M, Medina A. High-flow oxygen therapy in infants with bronchiolitis. N Engl J Med. 2018;378(25):2444. https://doi. org/10.1056/NEJMc1805312.

14. Mace AO, Gibbons J, Schultz A, Knight G, Martin AC. Humidified high-flow nasal cannula oxygen for bronchiolitis: should we go with the flow? Arch Dis Child. 2018;103(3):303. https://doi.org/10.1136/archdischild-2017-313950.

15. Sochet AA, McGee JA, October TW. Oral nutrition in children with bronchiolitis on high-flow nasal cannula is well tolerated. Hosp Pediatr. 2017;7(5):249255. https://doi.org/10.1542/hpeds.2016-0131. 